\title{
Hands-on physics displays for undergraduates
}

\section{Carl Akerlof}

Carl W. Akerlof, "Hands-on physics displays for undergraduates," Proc. SPIE 9289, 12th Education and Training in Optics and Photonics Conference, 92892W (17 July 2014); doi: 10.1117/12.2070782

SPIE Event: 12th Education and Training in Optics and Photonics Conference, 2013, Porto, Portugal 


\title{
Hands-on physics displays for undergraduates
}

\author{
Carl W. Akerlof \\ Physics Department, University of Michigan, Ann Arbor, Michigan, USA
}

\begin{abstract}
Initiated by Frank Oppenheimer in 1969, the Exploratorium in San Francisco has been the model for hands-on science museums throughout the world. The key idea has been to bring people with all levels of scientific background in contact with interesting and attractive exhibits that require the active participation of the visitor. Unfortunately, many science museums are now forced to cater primarily to very young audiences, often 8 years old or less, with predictable constraints on the intellectual depth of their exhibits. To counter this trend, the author has constructed several hands-on displays for the University of Michigan Physics Department that demonstrate: (1) magnetic levitation of pyrolytic graphite, (2) the varied magnetic induction effects in aluminum, copper and air, (3) chaotic motion of a double pendulum, (4) conservation of energy and momentum in a steel ball magnetic accelerator, (5) the diffraction pattern of red and green laser pointer beams created by CDs and DVDs, (6) a magnetic analog of the refraction of light at a dielectric boundary and (7) optical rotation of light in an aqueous fructose solution. Each of these exhibits can be constructed for something like $\$ 1000$ or less and are robust enough to withstand unsupervised public use. The dynamic behavior of these exhibits will be shown in accompanying video sequences.
\end{abstract}

The following story has a history that goes back quite a few years. In the late 70's, I was spending time at the Stanford Linear Accelerator Center accompanied by my family that included our two grade school children. Needless to say, we much enjoyed weekend excursions to all sorts of interesting sites in the Bay Area, especially the Exploratorium, an unusual science museum created by Frank Oppenheimer that opened in 1969. The notion that exhibits would be designed specifically for "hands-on" interactions was at that time quite revolutionary. This idea captivated a number of people everywhere including a friend in Ann Arbor, Cynthia Yao. With a core group of a few dozen people, Cynthia convinced the City of Ann Arbor to allow free use of an old firehouse. The Ann Arbor Hands-On Museum opened its doors to the public in 1982 and remains a thriving institution to this day.

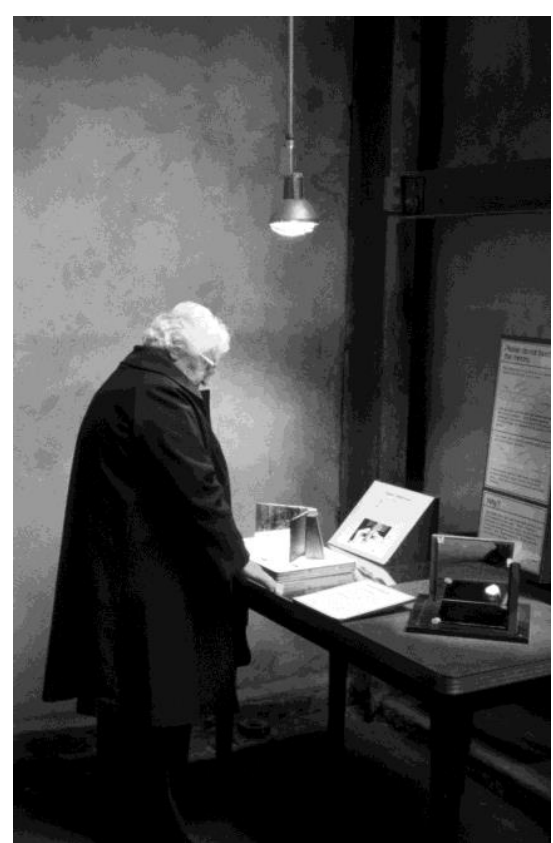

Figure 1a. Exploratorium ( 1977).

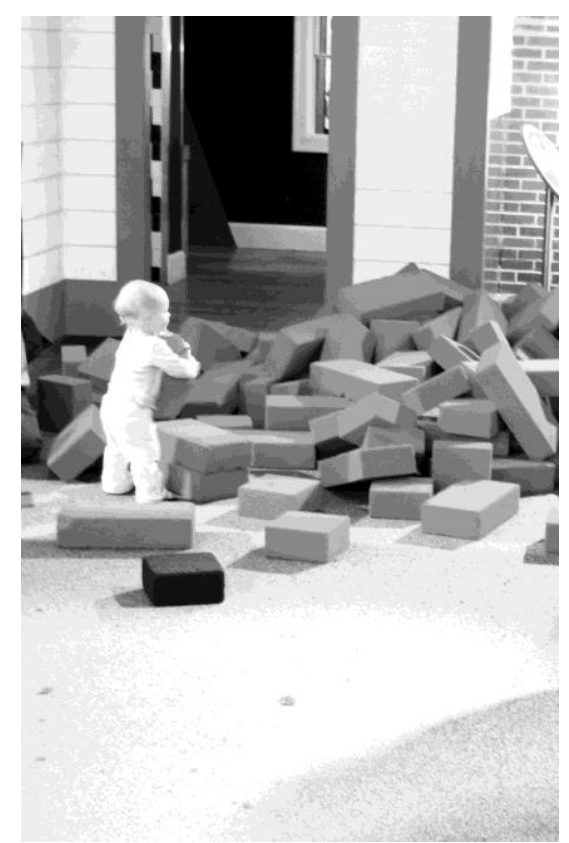

Figure 1b. Ann Arbor Hands-On Museum (2010).

12th Education and Training in Optics and Photonics Conference, edited by

Manuel F. P. C. Martins Costa, Mourad Zghal, Proc. of SPIE Vol. 9289, 92892W

(C) 2014 SPIE, OSA, IEEE, ICO · doi: 10.1117/12.2070782 
The original Exploratorium was guided by Oppenheimer's strong feelings that his creation must present interesting science with esthetically appealing exhibits. It was housed in the abandoned Palace of Fine Arts built for the 1915 Panama Pacific International Exposition in San Francisco which manifested the artistic charm of an airplane hangar. Frank was not concerned about the building - his focus was on the science exhibits. Most of us who helped start the Ann Arbor Hands-On Museum shared this same ethic. As our children grew up, I became only an occasional visitor to our Museum although I understood that more recent directors had grown oblivious to the intellectual challenges of maintaining a vibrant program. Then, about $2 \frac{1}{2}$ years ago, I was asked to serve on a museum advisory committee and the reality of contemporary science programs for the public became painfully clear as the director announced that the core audience was children no more than 8 years old. As graphic evidence, see the contrasts of two pairs of photographs of the Exploratorium and the Ann Arbor Museum taken about 30 years later. I hope that the differences do not need further elaboration.

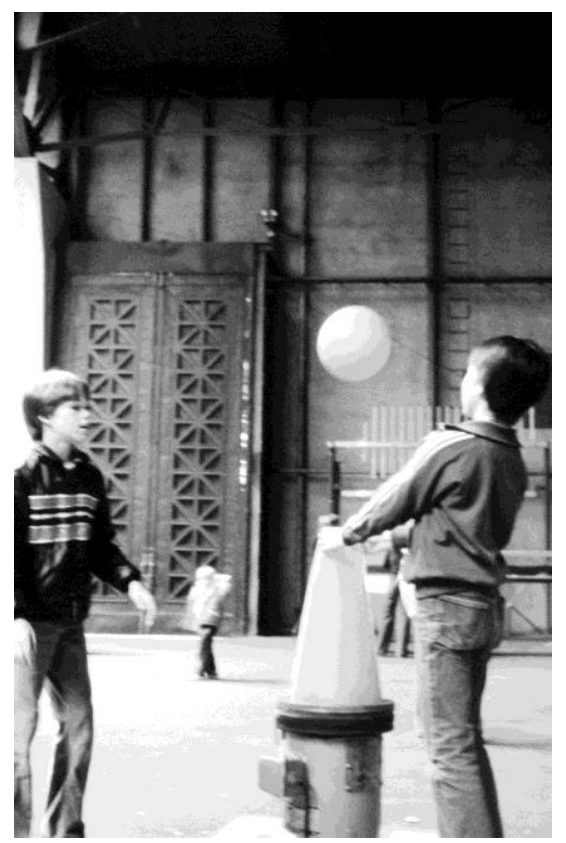

Figure 2a. Bernoulli effect exhibit at the Exploratorium ( 1977).

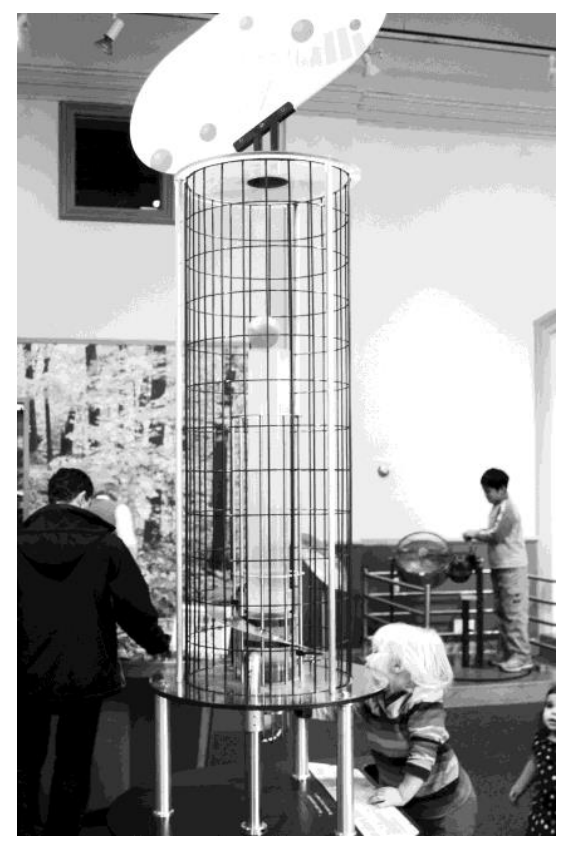

Figure 2b. Bernoulli effect exhibit at the Ann Arbor Hands-On Museum (2010).

A few months after this epiphany, I was asked to present a public lecture at the University of Michigan which I titled the Art of Physics. At the strong urging of the organizers for this talk, I brought along a number of cute physics demonstrations that I'd been assembling over a decade or so. Following my lecture, the audience got a chance to play with these gadgets, prompting me to consider rebuilding several of these items for a permanent display within our physics department. That is the main focus of this presentation.

Seven exhibits were designed and installed in a publically accessible $8^{\prime} \times 16^{\prime}$ hallway alcove on the second floor of our physics building. It is a bit removed from major student traffic but was accorded the blessings of the safety folks who worry about anything that might hinder movement in case of fire or other emergency. The list of exhibits is:

1. Magnetic levitation of pyrolytic graphite

2. Falling magnet demonstration of magnetic induction in aluminum and copper

3. Chaotic motion of the double pendulum

4. Steel ball linear accelerator

5. Light diffraction from optical disks

6. Electromechanical analog of optical refraction

7. Optical rotation in natural sugars 


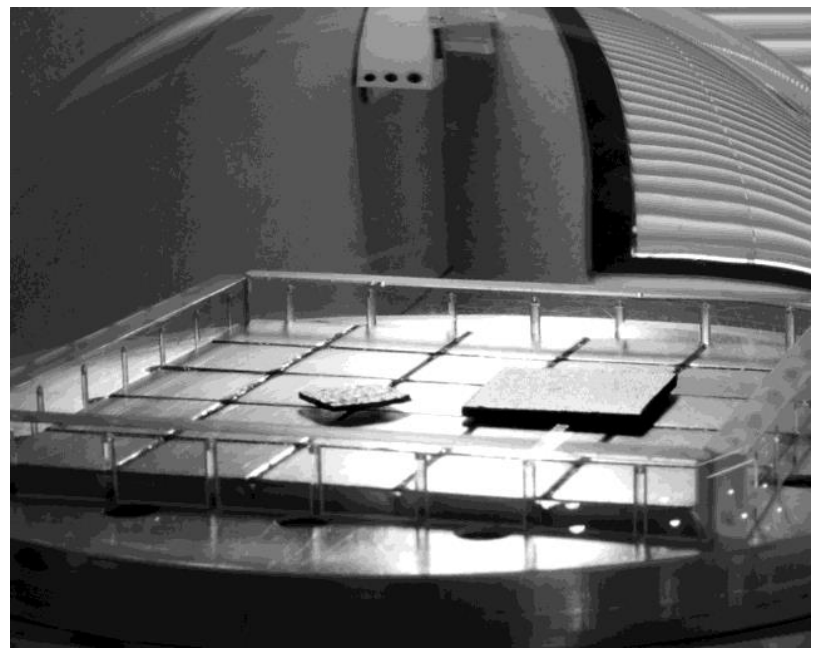

Figure 3a. Diamagnetic levitation of pyrolytic graphite.

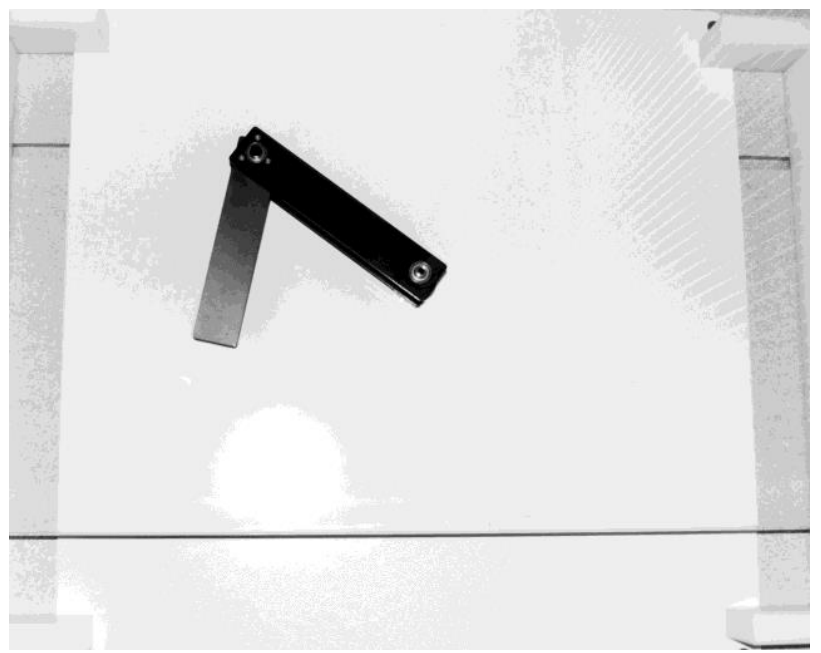

Figure 3c. Double pendulum in the middle of numerous chaotic gyrations.

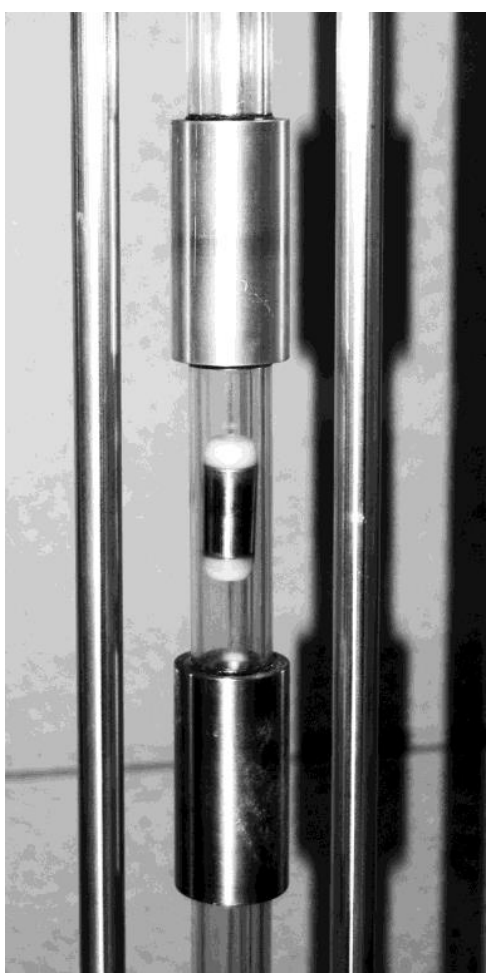

Figure 3b. Magnetic induction with a falling magnet.

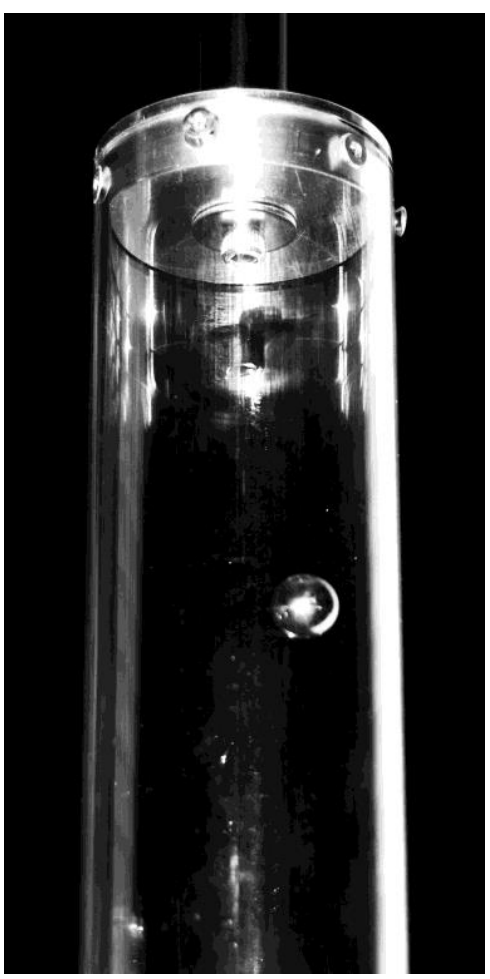

Figure 3d. Steel ball after acceleration by a linear array of magnets. 


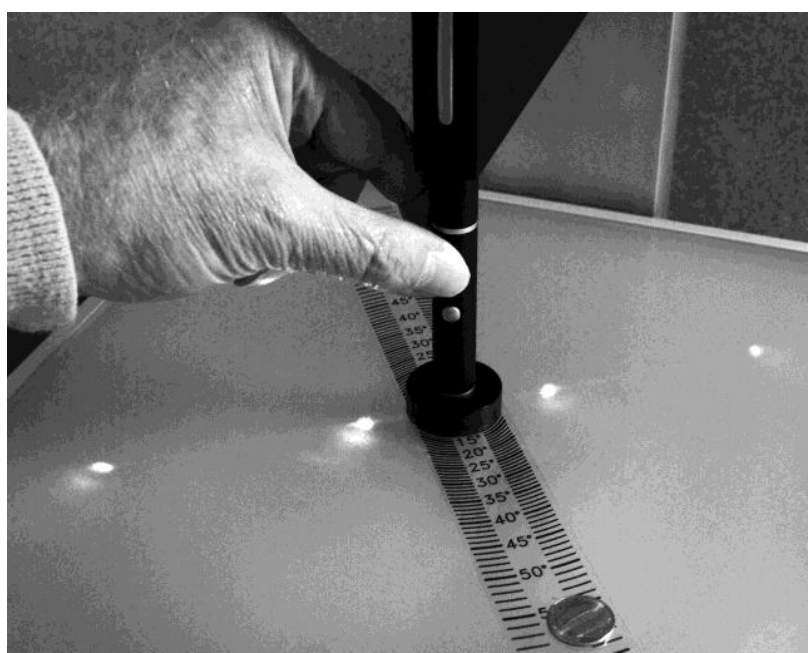

Figure 3e. Diffraction images of a laser pointer beam incident on a $\mathrm{CD}$

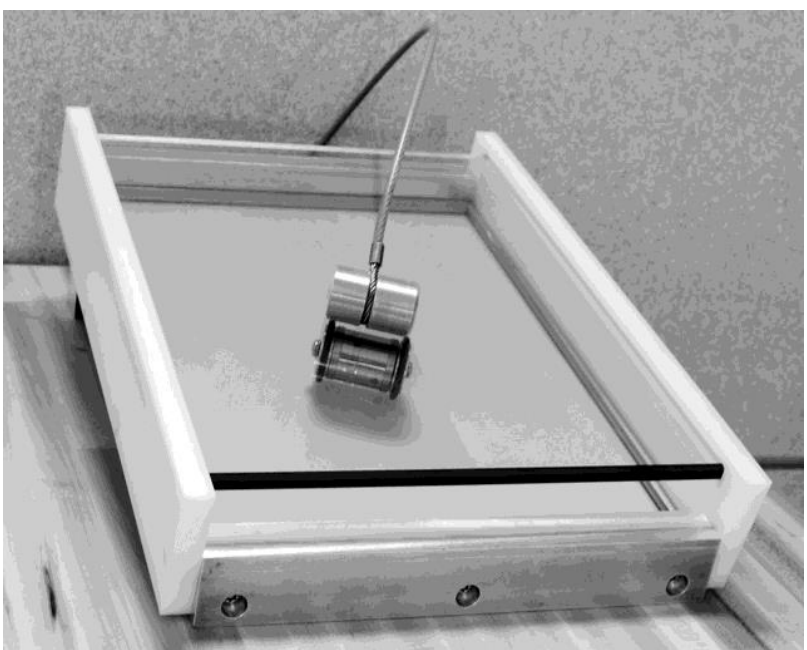

Figure 3f. Electromechanical analog of light refraction at a boundary.

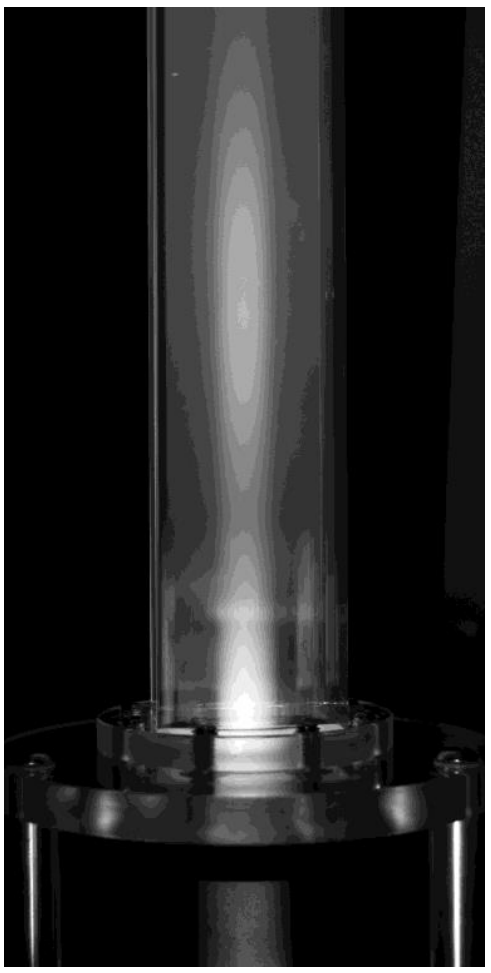

Figure 3g. Optical rotation of linearly polarized light in a column of fructose sugar solution.

All of these exhibits will be described in more detail in a sequence of video clips that explain the basic physics principles and show the behavior. A rough idea of what these look like can be obtained from Figures $3 \mathrm{a}-3 \mathrm{~g}$.

It has now been 2 years since these exhibits were first installed so it is appropriate to summarize our overall experience with this project. First of all, the design of the exhibits emphasized sturdy, robust construction. This has paid off in terms of essentially zero breakage and low maintenance. A rough estimate of the cost is about a $\$ 1000$ per exhibit with a reasonably large dispersion. To install these devices in a publically accessible space requires careful compliance 
with various safety and Americans with Disabilities Act (ADA) regulations. These strongly discourage use of AC power and forbid any loose parts. Any component of an exhibit that requires manual access must be no greater than 48 " from the floor $(122 \mathrm{~cm})$. These and various other boundary conditions were met without serious compromise of the original concepts. Student usage is not well documented but probably suffers from very low foot traffic in the current location. I hope that these seven exhibits contain various design features that could be usefully carried over to a variety of new display concepts.

Finally, I would like to conclude with some personal views about why such informal educational resources are important in our current culture. When I was a teenager, a significant fraction of my male cohorts tinkered with automobiles, learning all sorts of things about mechanical and electrical systems even if accompanied by a variety of mistakes along the way. However, symbolic manipulation was, at best, a dream. In the $21^{\text {st }}$ Century, this is completely reversed. It is basically impossible to alter a car in any substantial way whatsoever - a typical modern automobile contains over a dozen microprocessors with proprietary codes. Not only is the youthful pastime of "hotting up" cars illegal, it is technically more difficult than open heart surgery and less likely to succeed. On the other hand, my hardearned skills in learning to analytically integrate algebraic functions are irrelevant in the face of such symbolic computational aids as Mathematica. Our undergraduate students spend four years taking courses in mechanics, electromagnetism and optics that primarily deal with solving various differential equations. The concrete realities that the mathematics attempt to abstract are usually touched upon in the most superfluous way. In a world in which cellphone texting is the favored form of human communication, we need to remember that the physical world is deeper than mere symbolic processing, no matter how clever or complex. 